\title{
Mechanistic insights into differential requirement of receptor dimerization for oncogenic activation of mutant EGFR and its clinical perspective
}

\author{
Jeonghee Cho ${ }^{*}$ \\ Department of Nanobiomedical Science, Dankook University, Cheonan 31116, Korea
}

The epidermal growth factor receptor (EGFR), a member of the ErbB family (EGFR, ErbB2, ErbB3 and ErbB4), plays a crucial role in regulating various cellular responses such as proliferation, differentiation, and survival. As a result, aberrant activation of EGFR, mostly mediated through different classes of genomic alterations occurring within EGFR, is closely associated with the pathogenesis of numerous human cancers including lung adenocarcinoma, glioblastoma, and colorectal cancer. Thus, specific suppression of oncogenic activity of mutant EGFR with its targeted drugs has been routinely used in the clinic as a very effective anti-cancer strategy in treating a subset of tumors driven by such oncogenic EGFR mutants. However, the clinical efficacy of EGFR-targeted therapy does not last long due to several resistance mechanisms that emerge in the patients following the drug treatment. Thus, there is an urgent need for the development of novel therapeutic tactics specifically targeting mutant EGFR with the focus on the unique biological features of various mutant EGFR. Regarding this point, our review specifically emphasizes the recent findings about distinct requirements of receptor dimerization and autophosphorylation, which are critical steps for enzymatic activation of EGFR and signaling cascades, respectively, among wildtype and mutant EGFR and further discuss their clinical significance. In addition, the molecular mechanisms regulating EGFR dimerization and enzymatic activity by a key negative feedback inhibitor Mig6 as well as the clinical use for developing potential novel drugs targeting it are described in this review. [BMB Reports 2020; 53(3): 133-141]

\section{INTRODUCTION}

The ErbB family consists of four members including the epider-

*Corresponding author. Tel: +82-41-550-1172; Fax: +82-41-559-7839; E-mail: jeonghee.cho@dankook.ac.kr

https://doi.org/10.5483/BMBRep.2020.53.3.025

Received 3 February 2020

Keywords: cetuximab, Dimerization, EGFR, erlotinib, Mig6 mal growth factor receptor (EGFR), ErbB2, ErbB3, and ErbB4, and all of which are receptor tyrosine kinases (RTKs) $(1,2)$. Like the other RTKs, all four members of the ErbB family possess the key structural compositions as follows; extracellular ligand-binding domain, transmembrane domain, intracellular kinase domain, and carboxyl terminal (C-terminal) domain. Previous three-dimensional crystal structural analyses of ErbBs have provided in-depth mechanistic insights into how these domains coordinately contribute to structural rearrangements and overall receptor activation upon ligand stimulation $(3,4)$. As a consequence of the acquisition of enzymatic activity of the receptor, activated ErbBs initiate phosphorylating of numerous signaling molecules including the receptor itself on tyrosine residues within their C-terminal domains. In turn, these autophosporylated tyrosine residues of the receptor serve as a docking site for several intracellular adaptor proteins containing $\mathrm{SH} 2, \mathrm{SH} 3$ or PTB motifs, which subsequently trigger a plethora of signaling cascades such as RAS-RAF-MEK-MAPK and PI3K-AKT-mTRO, resulting in key biological effects including proliferation, migration, differentiation, and survival (5).

Considering the significant role of EGFR as a mitogenic inducer in cells, it is not surprising that the abnormal activation of EGFR is among very common oncogenic driving events in human cancer, resulting from various types of genomic alterations within EGFR (6). These genomic changes are mainly somatic EGFR mutations within regions of either the extracellular domain or the kinase domain, and EGFR amplification as found in several types of solid tumors (7-10). In addition, genomic deletions within exons encoding either the extracellular or C-terminal domain of EGFR have been identified in glioblastoma and lung adenocarcinoma, and their oncogenic potential was functionally characterized in vitro and in vivo (11-13).

One key question that has yet to be clearly addressed in the field of EGFR research is whether various oncogenic mutant EGFR are just a functional phenocopy of sustained wildtype EGFR activated by ligands or if these mutant EGFR have unique biological features such as distinct activation mechanisms and/or induction of differential signaling networks. Given that targeting mutant EGFR with specific inhibitors has been used as an effective clinical strategy in treating a subset of tumors

ISSN: 1976-670X (electronic edition)

Copyright (C) 2020 by the The Korean Society for Biochemistry and Molecular Biology

(c) This is an open-access article distributed under the terms of the Creative Commons Attribution Non-Commercial License (http://creativecommons.org/licenses/by-nc/4.0) which permits unrestricted non-commercial use, distribution, and reproduction in any medium, provided the original work is properly cited. 
harboring such mutations, a detailed elucidation of the biological aspects specific to mutant EGFR would provide invaluable insight on the designing of novel therapeutic tactics.

In this review, we focus on the distinct mechanisms leading to receptor activation between wildtype and mutant EGFR, especially on the requirement of dimerization and also the clinical potential of utilizing this feature in designing novel EGFR-targeted drugs.

\section{EGFR ACTIVATION BY RECEPTOR DIMERIZATION}

\section{Ligand-mediated extracellular domain dimerization}

Full enzymatic activation of EGFR is achieved following a series of conformational changes throughout the receptor, initiated by ligand-mediated receptor dimerization. Seven ligands are known to bind to EGFR and the other ErbB family members, which include the epidermal growth factor (EGF), transforming growth factor- $\alpha$ (TNF- $\alpha$ ), epiregulin (EPR), heparinbinding EGF-like growth factor (HB-EGF) and amphiregulin (AR) $(14,15)$. The contribution of these ligands in the rearrangement of extracellular domain priming for EGFR homo- or heterodimerization with the other ErbB family members have been well characterized by numerous crystal structure studies (16-19). The extracellular regions of EGFR comprise of two homologous ligand binding domains (domains I and III) and two cystine rich domains (domains II and IV). In the absence of ligands, EGFR exists as an inactive tethered conformation in which domains II and IV interact with and occlude domain II "dimerization arm" involved in receptor dimerization. Notably, based on the current proposed model, this tethered state is preferred in the absence of ligands, but it is not the only form and multiple untethered conformations could exist in a dynamic equilibrium. The binding of ligands to domain I and III stabilizes the un-tethered receptors in a dimerization-competent extended conformation, consequently driving the overall equilibrium shift from an inactive tethered monomer towards the active dimeric forms (Fig. 1A) (20). Thus, it is well accepted that the stabilization of dimerization arm exposed monomeric forms led by various ligand-mediated extracellular domain rearrangement could be a crucial step leading to receptor dimerization.

\section{Asymmetric dimerization of kinase domains}

There is no doubt that one of the breakthrough studies in the field of EGFR biology is the detailed crystal structure-based mechanistic elucidation of asymmetric dimerization of kinase domains-mediated EGFR activation $(21,22)$. This study revealed that two interfaces between the $\mathrm{N}$-lobe of one kinase domain, termed "receiver", and C-lobe of the other kinase domain, termed "activator", interact in an asymmetric manner. Consequently, the activator monomer induces the conformational changes of the N-lobe of the receiver monomer, similar to cyclin-induced activation of cyclin-dependent kinases, resulting in the enzymatic activation of the receiver monomer in a

\section{Differential requirement of EGFR dimerization for activation}

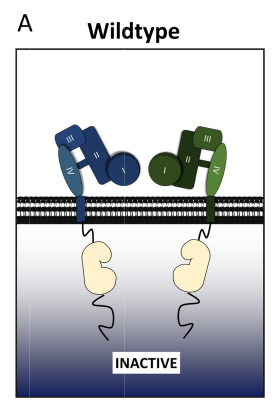

B Dimerization-dependent mutant group

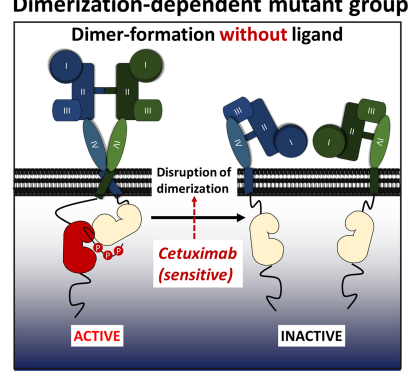

Ligand 1 Cetuximab
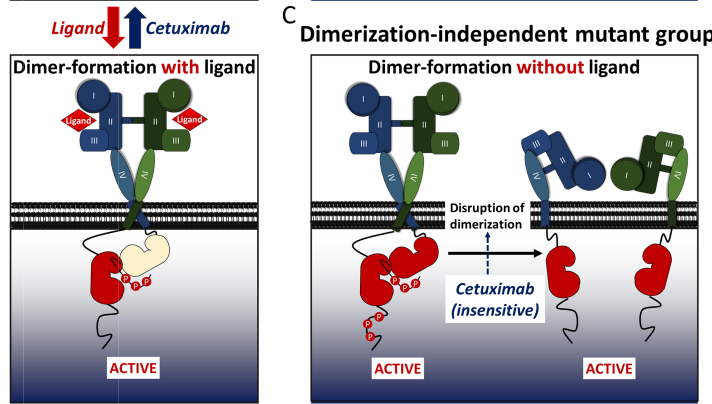

ation-independent mutant group

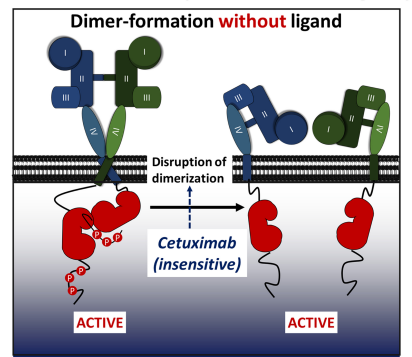

Fig. 1. Proposed model of dimerization requirements for recepto activation by wildtype EGFR and oncogenic mutants (adapted from Cho et al., 2013). (A) Activation of wildtype EGFR is initiated by ligand-induced extracellular dimerization of the receptor, which in turn direct "outside-in" asymmetric dimerization of the $\mathrm{N}$ - and C-lobes in the kinase domain, leading to enzymatic activation of the receiver monomer and subsequent autophosphorylation on the C-terminal tail of the activator monomer. Therefore, full enzymatic activation and consequent signaling cascade of the wildtype EGFR are tightly dependent on all sequential events of ligand binding, dimerization and autophosphorylation. (B) A group of EGFR mutants including the L858R, most colon cancer-derived and C-terminal truncation EGFR mutants are able to form constitutive asymmetric dimerization irrespective of ligand binding, which in turn promotes "inside-out" extracellular dimerization; ligand increases dimerization but is not required. Interruption of dimerization impairs the oncogenic activity of these types of mutant EGFR because the receiver monomer is no longer active. Thus, mutant EGFR belong to this group is sensitive to EGFR-directed $m A$ s such as cetuximab. (C) In contrast, a subset of mutant EGFR including the Ex19Del and Ex20Ins mutants and the L858R/T790M double mutants similarly undergo dimerization in the absence of ligand, but do not require dimerization for their activity. These mutant forms of EGFR acquire the oncogenic activity in dimerization-independent manner so that these mutants are refractory to EGFR mAbs.

dimer (Fig. 1A) $(22,23)$. The functional significance of this finding was further validated by several subsequent studies $(24,25)$. For example, it was shown that the disruption of asymmetric dimerization through substitution mutations at the dimerization interface, such as L704N (receiver-impairing mutation) in the N-lobe and 1941R (activator-impairing mutation) in the C-lobe, abolished ligand-induced EGFR activation and consequent cellular transformation $(22,24,25)$. In addition, the co-expression of receiver-impaired and activator-impaired 
EGFR mutants can rescue EGFR activation through a subset of reestablished asymmetric dimerization between the intact $\mathrm{C}$-lobe and the intact $\mathrm{N}$-lobe of the respective EGFR mutants (25). Also, compelling evidence from numerous functional and structural studies support the current model that enzymatic activation of EGFR is mediated by the ligand-mediated extracellular dimerization of EGFR followed by the asymmetric dimerization of the kinase domains of two monomers in an allosteric manner (24-27). Thus, the coordination of two distinct dimerization allosterically achieved through conformational changes of the receptor may function as critical steps in inducing enzymatic activation of EGFR. Notably, several regulatory mechanisms tightly modulating these processes have been identified as essential cellular devices for controlling receptor activation as well as for preventing unnecessary EGFR activation.

\section{GENOMIC ALTERATIONS OF EGFR AND MECHANISTIC INSIGHTS INTO ONCOGENIC ACTIVATION}

\section{Various types of EGFR mutations in cancer}

Several different classes of genomic mutations within EGFR have been found in various cancer types, including lung adenocarcinoma, glioblastoma (GBM), and colorectal adenocarcinoma (Fig. 2) $(7,12,24,28-30)$. In addition, numerous structural and functional studies have shown that a subset of patient-derived EGFR mutations are directly associated with ligand-independent receptor activation and dysregulation of the EGFR signaling cascade, consequently resulting in cellular transformation (3133). In lung adenocarcinoma, the most dominant mutations are within the kinase domain of EGFR, including L858R in exon 21 and various small in-frame deletions in exon 19 (Ex19Del), with these genomic alterations making up 90\% of EGFR mutations identified from lung adenocarcinoma (Fig. 2) (34). In particular, these two prevalent types of somatic mutations have clinical significance because lung tumors harboring such mutations are highly sensitive to EGFR kinase targeted inhibitors such as gefitinib and erlotinib (35). Thus, the EGFR mutation status in lung cancer patients serves as a crucial genomic determinant and biomarker for clinical outcomes with EGFR-directed therapy. In GBM, EGFR alterations were frequently identified as either intragenic deletions between exons 2-7 (denoted as EGFR vlII), exons 14-15 (denoted as EGFRvII) or somatic alterations within the extracellular domain of the receptor, but lung cancer prevalent kinase domain mutations are relatively rare (Fig. 2) $(7,36)$. Furthermore, recent genomic studies identified several additional intragenic deletions of exons encoding the EGFR C-terminal domain in GBM, also found in lung adenocarcinoma (Fig. 2) $(7,12)$. Subsequent studies have showed that these resulting C-terminal truncation mutants retain the ability to induce oncogenic transformation and tumorigenesis $(12,27)$. Notably, in vitro and in vivo experiments clearly demonstrated that therapeutic anti-EGFR monoclonal antibodies such as cetuximab are highly effective against GBM-derived C-terminal deletion EGFR variants $(12,27)$. However, the clinical efficacy of cetuximab-directed therapy for GBM patients harboring such mutations has not been proven. In colorectal cancer, according to the public genomic databases (e.g. cBioportal), EGFR mutations have been known to be very rare $(\sim 3 \%)$.

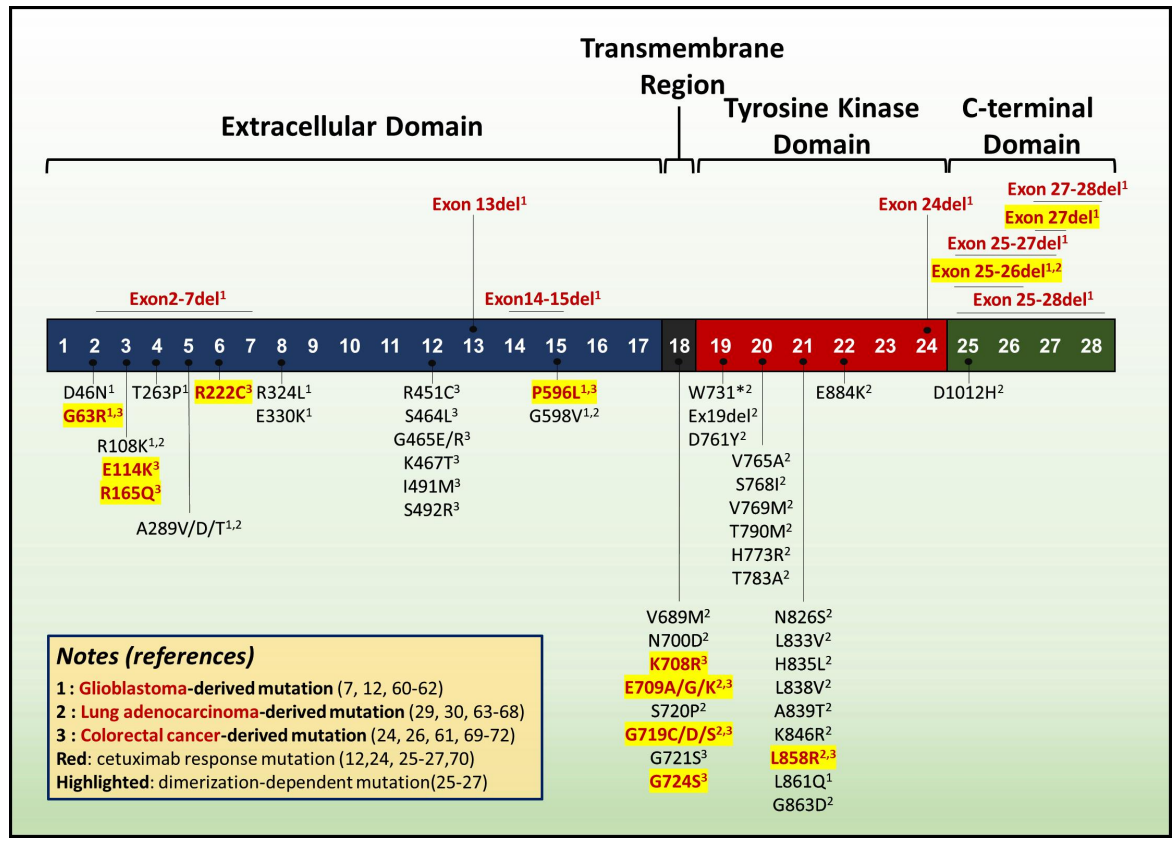

Fig. 2. A subset of major EGFR mutations derived from cancer-patients are presented according to the location of each exon. Confirmed dimerization-dependent mutations and cetuximab-sensitive mutations are specifically indicated in the schematic diagram as shown in notes. 
However, very recent systematic functional and biochemical studies with recurrent EGFR mutations selected from public genomic datasets of colorectal cancer revealed that a subset of colon cancer-derived EGFR mutants function as a strong oncogenic driver in a ligand-independent manner (Fig. 2) (24, 26). This result suggests that although EGFR mutations are not as common as other cancer types, these somatic alterations may contribute to the pathogenesis of colorectal cancer. Significantly, the oncogenic potential of these EGFR mutants is efficiently inhibited by cetuximab or panitumumab in vivo and in vitro $(24,26)$. Thus, further exploration is needed as to whether these EGFR mutations can serve as clinically beneficial genomic biomarkers for anti-EGFR antibodies in colon cancer patients with such mutations as shown in lung adenocarcinoma.

\section{Distinct requirement of dimerization for oncogenic activation of mutant EGFR}

It is well characterized that various EGFR oncogenic mutations induce constitutive activation of enzymes that are not dependent on ligands $(12,24,31)$. Given that asymmetric dimerization of kinase domains directed by ligand-mediated extracellular dimerization is a critical step for canonical enzymatic activation of wild-type EGFR (22), it is of significance to examine whether ligand-independent EGFR mutants still require asymmetric dimerization for constitutive receptor activation leading to oncogenic cellular transformation. Several biochemical and functional studies have been reported regarding these issues. A key experimental strategy used in these studies was to functionally evaluate the dependency of dimerization in the oncogenic potential of various cancer-derived mutant EGFR by characterizing the dimerization-disrupted compound EGFR mutants with either a receiver-impairing mutation (L704N) and/or an activator-impairing mutation (I941R) using NIH-3T3 cells, which express low to undetectable levels of endogenous EGFR. These dimerization-incompetent EGFR mutant-expressing cells were then assayed for enzymatic activity of EGFR mutants and their ability to grow in soft agar. In this system, the EGFR activation and transforming ability of dimerization-dependent mutants is predicted to be abolished by cis mutation of L704 or 1941 (22). Thus, this experiment can be used to determine whether various EGFR mutants can be activated and induce cellular transformation in a dimerization-dependent or -independent fashion. Based on the results from these approaches, interestingly, two distinct requirements of dimerization for oncogenic activation were shown to exist among various mutant EGFR. While the lung cancer-derived L858R, GBMderived C-terminal deletions and most of colon cancer-derived EGFR mutants require dimerization for their oncogenic activation (Fig. 1B), a subset of lung-cancer derived mutants such as Ex19Del, Ex20Ins, and L858R/T790M EGFR mutants do not depend on dimerization for constitutive receptor activation (Fig. 1C) (24-27). These results suggest that a key molecular mechanism leading to oncogenic activation of L858R, C-terminal deletions and colon cancer-derived EGFR mutants may be linked to the acquisition of the ability to form ligand-independent constitutive dimerization on the cell surface. In contrast, the other group including Ex19Del, Ex20Ins, and L858R/T790M EGFR mutants appears to acquire oncogenic activation irrespective of these dimerization (25). Thus, two distinct classes of mutant EGFR exist based on the dependence on dimerization for oncogenic activation. Also, it has been shown that the difference in dimerization requirement among mutant EGFR is also associated with the induction of various downstream signaling networks leading to cellular transformation. For example, the addition of dimerization-impairing cis mutations, L704N and 1941R, in the dimerization-dependent EGFR mutants significantly reduced their ability to promote colony formation of $\mathrm{NIH}-3 \mathrm{~T} 3$ cells upon retroviral transduction (24-27). In contrast, introduction of the same dimerizationdisruption mutations into the dimerization-independent group of EGFR mutants had little effect on their ability to induce colony formation under the same experimental condition, suggesting that this group of mutants can indeed result in cellular transformation irrespective of dimerization (25).

Notably, these unexpected results are further supported by a recent report showing that C-terminal autophosphorylation of dimerization-independent mutant EGFR such as Ex20lns is not required for oncogenic transformation (37). One proposed explanation for this result is that Gab1/2, Shc1 and Bcar1 adaptors may interact in a mutant-EGFR-specific manner and function as crucial factors in mediating constitutive oncogenic activation of various signaling pathways independently of asymmetric dimerization and C-terminal phosphorylation (37).

In summary, EGFR mutants seem to become active via different paths, which can be achieved in a dimerizationindependent manner and also lead to activation of signaling networks without C-terminal autophosphorylation. The more detailed molecular mechanisms and its functional significance of these non-canonical biological features of mutant EGFR need to be further explored in future studies.

\section{REGULATION OF EGFR DIMERIZATION AS NEGATIVE FEEDBACK MECHANISM}

\section{Key effectors associated with negative regulation of active EGFR}

Deactivation of ligand-stimulated active EGFR is temporally and spatially regulated via numerous mechanisms to prevent unnecessarily sustained cellular responses, closely associated with the pathogenesis of various diseases including cancer. These negative regulatory mechanisms of EGFR can be classified into two major modes of action which includes degradation of ligand-bound active EGF receptors, and inactivation of enzymatic activity of the receptor (38). Collectively, these processes are mediated by tightly coordinated actions of a plethora of proteins which effectively impede the function of active EGFR as well as its downstream signaling cascade. Specifically, the degradation of active EGFR is primed via the 
ubiquitination of the receptor resulting from the direct binding of Cbl, E3 ubiquitin ligase, to autophosphorylated EGFR at tyrosine 1069, which subsequently induce the sorting of the receptor to lysosomal fusion for its degradation (39). In addition, four negative feedback inhibitors, LRIG, Mig6 (also called RALT) and SOCS4/5, transcriptionally induced by activated ErbB signaling networks, have been identified as the key regulators of ErbB activity including EGFR (40). Among them, the functional roles of Mig6 as a negative effector of EGFR are the most intensively characterized (41) and the molecular mechanisms underlying Mig6-mediated negative regulation of EGFR is specifically discussed below based on recent findings from several structural and functional studies.

\section{Mig6 functions as a key negative regulator of EGFR}

Mitogen-inducible gene 6 (Mig6), also known as RALT, is a negative feedback inhibitor of EGFR and other ErbB family members. Mig6 expression is transcriptionally induced by hormones, growth factors, and various stress stimuli via the Ras-Raf-ERK signaling pathway $(42,43)$. By complexing with EGFR mediating revolutionary conserved domains (segment 1 and 2) (44), Mig6 effectively inhibits EGFR activation and its downstream signaling pathways via three proposed mechanisms in an orchestrated manner. First, Mig6 binds to the C-lobe of the active EGFR kinase domain through segment 1 and blocks the asymmetric dimer formation with the $\mathrm{N}$-lobe of the other EGFR monomer, required for full enzymatic activation of EGFR as described above (Fig. 3) (45). Second, phosphorylated Mig6 at segment 2 binds strongly to the EGFR kinase domain and directly suppresses its catalytic activity in a peptide substrate competitive manner (Fig. 3) (46). In addition, Mig6 can complex with EGFR and induce its internalization and degradation $(47,48)$. Such coordinated actions seem to render Mig6 as a tight negative regulator of EGFR and its signaling circuits to prevent aberrant EGFR activation leading to cellular transformation. Consistent with this notion, the loss of Errfi1 (ERBB receptor feedback inhibitor 1, official gene name of Mig6) in mice was shown to induce a high incidence of neoplastic lesions caused by abnormal activation of EGFR signaling (49). Furthermore, frequent focal deletions of ERRFI1 were identified from the analysis of 1,057 gliomas supporting the fact that the loss of Mig6 is a key driving force of human cancer (46).

\section{CLINICAL SIGNIFICANCE OF BLOCKAGE OF EGFR DIMERIZATION}

\section{EGFR targeted monoclonal antibody}

Several EGFR-directed therapeutic monoclonal antibodies $(\mathrm{mAb})$ such as cetuximab and panitumumab are effective in the treatment of a subset of tumors such as colon cancer (50, 51). Although the mechanisms are unclear, previous structural studies suggested that one proposed pharmacological mode of action of these EGFR-targeted Abs is to interfere with EGFR
Mig6-mediated negative feedback mechanisms regulating EGFR activity

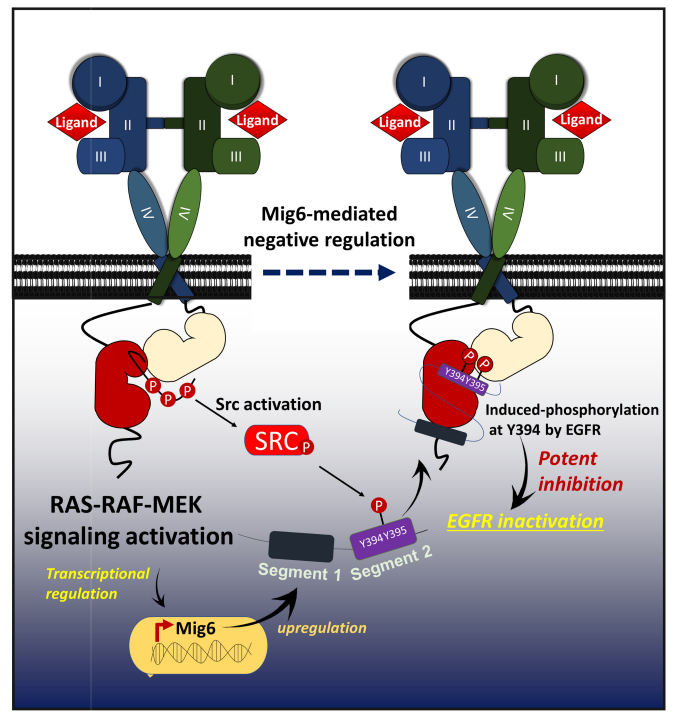

Fig. 3. Model for mechanism of feedback inhibition of EGFR by Mig6 (adapted from Park et al., 2015). Activated EGFR upregulates the expression of Mig6 via the Ras-RAF-Map kinase signaling pathway. Mig6 segment 1 binds to dimerization interface of EGFR monomer and block the further asymmetric dimerization. In addition, Src activated by EGFR phosphorylates Mig6 on Y395 in segment 2. This phospho-segment 2 of Mig6 interact with EGFR and is further phosphorylated on Y394, which renders it a potent inhibitor of EGFR. After phosphorylation, segment 2 bound to EGFR rearranges to block the peptide-substrate binding cleft. A single Mig6 protein should be sufficient to inactivate the wildtype receptor, as only one subunit in the asymmetric dimer is active and able to phosphorylate Mig6.

dimerization via specifically binding to the ligand interacting region of the receptor so that the drugs block the enzymatic activation of EGFR (20). As discussed above, given that a subset of oncogenic EGFR mutants such as exon 19 deletion and exon 20 insertion mutants are oncogenically active irrespective of receptor dimerization, it is postulated that antibodies directed at the "upstream" extracellular domain may be ineffective against these dimerization-independent oncogenic mutant EGFR. Several recent in vivo and in vitro studies provided compelling evidence to support this hypothesis, showing that there is a close relationship between dimerization dependency and EGFR-directed $\mathrm{mAb}$ responses among mutant EGFR (2427). For example, mouse lung tumors formed by dimerizationdependent L858R and G719S EGFR mutants are dramatically reduced by cetuximab treatment, whereas tumors induced by dimerization-independent mutant EGFR such as the exon 20 insertion mutant are resistant to cetuximab (Fig. 1B, 1C, and 2) (25). Similarly, the oncogenic activity of GBM-derived C-terminal deletion mutants, caused by their constitutive dimerization, is efficiently inhibited by cetuximab in vivo and in vitro (27). Furthermore, recent reports demonstrated that all 
dimerization-dependent colorectal cancer-derived EGFR have a dramatic response to cetuximab and panitumumab (Fig. 1B and 2) $(24,26)$. Taken together, these data suggest that ligandindependent constitutive receptor dimerization caused by somatic mutations within EGFR is a key molecular mechanism leading to oncogenic activation of EGFR (52) and disruption of dimerization may be among the pharmacological mechanisms of EGFR-targeted mAbs. Thus, it was proposed that the requirement of dimerization for oncogenic activation among mutant EGFR may be a crucial predictive factor of clinical response to cetuximab, as a close correlation exists between dimerization dependency and its pharmacological effects. This hypothesis needs to be further explored in future studies in clinical settings.

\section{Mig6-based dimerization inhibition}

The breakthrough findings of the key molecular mechanism underlying Mig6-mediated negative feedback regulation of EGFR was conducted through X-ray crystal-based structural studies followed by elaborate biochemical characterization (45). These studies revealed that Mig6 strongly interacts with EGFR in the asymmetric dimerization interface of EGFR kinase domain C-lobe and $\mathrm{N}$-lobe regions. Also, this interaction is mediated by a specific domain of Mig6 known as segment 1 (S1), one of two evolutionary conserved regions within Mig6 (45). Given that EGFR asymmetric dimerization is required for full enzymatic activation of EGFR (22), the binding of Mig6 S1 to EGFR abrogates the formation of intact dimerization of EGFR and consequently blocks its activation (Fig. 3). Mig6 also appears to prevent asymmetric dimerization among the other ErbB family members via the same interacting mechanism (45). Thus, the preclusion of asymmetric dimerization by Mig6 $\mathrm{S} 1$ is a key negative regulatory mechanism among ErbB members.

One puzzling observation not clearly addressed in the original structural analysis was the functional significance of the other interaction of Mig6 at the substrate binding site of EGFR, mediated by Mig6 segment 2 (S2) (45). Recent additional crystal structural, biochemical and functional studies demonstrated the role of Mig6 S2-mediated interaction in inhibiting EGFR activation and identified the detailed molecular mechanism of how Mig6 S2 contributes to this process (46, 53). According to these findings, Mig6 remains an incomplete inhibitor of EGFR until a series of posttranslational modifications occurs in segment 2; Mig6 undergoes tyrosine phosphorylation at 395 in S2 region by Src, which in turn becomes a suitable substrate of EGFR (46). Next, additional phosphorylation is induced by EGFR in Mig6 at tyrosine 394, which dramatically increases the binding affinity of Mig6 to the EGFR active site and consequently, Mig6 becomes a potential competitive inhibitor blocking other substrate access to EGFR (Fig. 3) (46). The functional significance of these events was further validated using in vitro models. While phosphorylation-competent Mig6 effectively prevents EGFR mutants from causing oncogenic transformations, this was not the case with
Mig6 Y394F/Y395F mutants under the same experimental conditions (46).

In conclusion, Mig6 negatively regulates EGFR and its signaling pathways through several distinct mechanisms; blockage of EGFR asymmetric dimerization by the S1 region and potential competitive inhibition of substrate access by the phosphorylated S2 region. This signaling circuit-based regulatory mechanism renders Mig6 effective in selectively targeting active EGFR.

\section{PERSPECTIVE}

Despite the proven effectiveness of EGFR-directed therapy in treating a subset of tumors driven by aberrant EGFR, its clinical efficacy is limited due to various resistance mechanisms such as additional T790M secondary mutation within EGFR emerging among patients following treatment of these drugs (54). Currently, numerous efforts have been made to generate additional effective targeted drugs to overcome such resistance and a number of FDA-approved drugs are already used in clinics (55). However, most next generation drugs are also based on ATP-analogs and thus remain highly susceptible to developing inevitable resistance (56). Considering that dimerization of EGFR is a critical biological step required for its enzymatic activation, an ideal approach may be to take advantage of these features in developing novel EGFR-targeted drugs (57). Regarding this point, Mig6 segment 1-based peptides specifically targeting dimers can be considered as a novel potential drug particularly for dimerization-dependent mutant EGFR. In addition, given that Mig6 can effectively block the oncogenic activity of most mutant EGFR including T790M mutant (46), a Mig6 394/395 phospho-peptides based drug design can also be adopted as an attractive approach to overcome current drug resistance. Recent reports have shown that small peptides encompassing Y394/Y395 can effectively bind to EGFR and block the enzymatic activity of EGFR $(58,59)$. The clinical significance of these peptides as a potential EGFR-directed drug needs to be further investigated in the future.

\section{ACKNOWLEDGEMENTS}

These works were in part supported by the National Research Foundation of Korea (NRF) grant funded by the Korea government (MEST) (NRF-2016R1A2B2011100, NRF-2019R1H1A 2079880 and NRF-2019R1A4A1028268).

\section{CONFLICTS OF INTEREST}

The author has no conflicting interests.

\section{REFERENCES}

1. Olayioye MA, Neve RM, Lane HA and Hynes NE (2000) The ErbB signaling network: receptor heterodimerization 
in development and cancer. EMBO J 19, 3159-3167

2. Yarden Y and Pines G (2012) The ERBB network: at last, cancer therapy meets systems biology. Nat Rev Cancer 12, 553-563

3. Schlessinger J and Lemmon MA (2006) Nuclear signaling by receptor tyrosine kinases: the first robin of spring. Cell $127,45-48$

4. Burgess AW, Cho HS, Eigenbrot C et al (2003) An open-and-shut case? Recent insights into the activation of EGF/ErbB receptors. Mol Cell 12, 541-552

5. Schlessinger J (2000) Cell signaling by receptor tyrosine kinases. Cell 103, 211-225

6. Sharma SV, Bell DW, Settleman J and Haber DA (2007) Epidermal growth factor receptor mutations in lung cancer. Nat Rev Cancer 7, 169-181

7. Lee JC, Vivanco I, Beroukhim R et al (2006) Epidermal growth factor receptor activation in glioblastoma through novel missense mutations in the extracellular domain. PLoS Med 3, e485

8. Castellanos E, Feld E and Horn L (2017) Driven by mutations: The predictive value of mutation subtype in EGFR-Mutated non-small cell lung cancer. J Thorac Oncol $12,612-623$

9. Hirono Y, Tsugawa K, Fushida S et al (1995) Amplification of epidermal growth factor receptor gene and its relationship to survival in human gastric cancer. Oncology 52, 182-188

10. al-Kasspooles $\mathrm{M}$, Moore $\mathrm{JH}$, Orringer MB and Beer DG (1993) Amplification and over-expression of the EGFR and erbB-2 genes in human esophageal adenocarcinomas. Int J Cancer 54, 213-219

11. Gan HK, Cvrljevic AN and Johns TG (2013) The epidermal growth factor receptor variant III (EGFRvIII): where wild things are altered. FEBS J 280, 5350-5370

12. Cho J, Pastorino S, Zeng Q et al (2011) Glioblastomaderived epidermal growth factor receptor carboxyl-terminal deletion mutants are transforming and are sensitive to EGFR-directed therapies. Cancer Res 71, 7587-7596

13. Imielinski M, Berger AH, Hammerman PS et al (2012) Mapping the hallmarks of lung adenocarcinoma with massively parallel sequencing. Cell 150, 1107-1120

14. Hynes NE and Lane HA (2005) ERBB receptors and cancer: the complexity of targeted inhibitors. Nat Rev Cancer 5, 341-354

15. Harris RC, Chung E and Coffey RJ (2003) EGF receptor ligands. Exp Cell Res 284, 2-13

16. Garrett TP, McKern NM, Lou M et al (2002) Crystal structure of a truncated epidermal growth factor receptor extracellular domain bound to transforming growth factor alpha. Cell 110, 763-773

17. Ogiso H, Ishitani $R$, Nureki $O$ et al (2002) Crystal structure of the complex of human epidermal growth factor and receptor extracellular domains. Cell 110, 775-787

18. Cho HS and Leahy DJ (2002) Structure of the extracellular region of HER3 reveals an interdomain tether. Science 297, 1330-1333

19. Cho HS, Mason K, Ramyar KX et al (2003) Structure of the extracellular region of HER2 alone and in complex with the Herceptin Fab. Nature 421, 756-760
20. Ferguson KM (2008) Structure-based view of epidermal growth factor receptor regulation. Annu Rev Biophys 37, 353-373

21. Groenen LC, Walker F, Burgess AW and Treutlein HR (1997) A model for the activation of the epidermal growth factor receptor kinase involvement of an asymmetric dimer? Biochemistry 36, 3826-3836

22. Zhang X, Gureasko J, Shen K, Cole PA and Kuriyan (2006) An allosteric mechanism for activation of the kinase domain of epidermal growth factor receptor. Cell $125,1137-1149$

23. De Bondt HL, Rosenblatt J, Jancarik J, Jones HD, Morgan DO and Kim SH (1993) Crystal structure of cyclin-dependent kinase 2. Nature 363, 595-602

24. Cho J, Bass AJ, Lawrence MS et al (2014) Colon cancerderived oncogenic EGFR G724S mutant identified by whole genome sequence analysis is dependent on asymmetric dimerization and sensitive to cetuximab. Mol Cancer 13, 141

25. Cho J, Chen L, Sangji N et al (2013) Cetuximab response of lung cancer-derived EGF receptor mutants is associated with asymmetric dimerization. Cancer Res 73, 6770-6779

26. Kim N, Cho D, Kim H et al (2020) Colorectal adenocarcinoma-derived EGFR mutants are oncogenic and sensitive to EGFR-targeted monoclonal antibodies, cetuximab and panitumumab. Int J Cancer 146, 2194-2200

27. Park AK, Francis JM, Park WY, Park JO and Cho J (2015) Constitutive asymmetric dimerization drives oncogenic activation of epidermal growth factor receptor carboxylterminal deletion mutants. Oncotarget 6, 8839-8850

28. Lynch TJ, Bell DW, Sordella R et al (2004) Activating mutations in the epidermal growth factor receptor underlying responsiveness of non-small-cell lung cancer to gefitinib. N Engl J Med 350, 2129-2139

29. Paez JG, Janne PA, Lee JC et al (2004) EGFR mutations in lung cancer: correlation with clinical response to gefitinib therapy. Science 304, 1497-1500

30. Pao W, Miller V, Zakowski M et al (2004) EGF receptor gene mutations are common in lung cancers from "never smokers" and are associated with sensitivity of tumors to gefitinib and erlotinib. Proc Natl Acad Sci U S A 101, 13306-13311

31. Greulich H, Chen TH, Feng W et al (2005) Oncogenic transformation by inhibitor-sensitive and -resistant EGFR mutants. PLoS Med 2, e313

32. Yun $\mathrm{CH}$, Boggon TJ, Li Y et al (2007) Structures of lung cancer-derived EGFR mutants and inhibitor complexes: mechanism of activation and insights into differential inhibitor sensitivity. Cancer Cell 11, 217-227

33. Guo G, Gong K, Wohlfeld B, Hatanpaa KJ, Zhao D and Habib AA (2015) Ligand-Independent EGFR Signaling. Cancer Res 75, 3436-3441

34. Chan SK, Gullick WJ and Hill ME (2006) Mutations of the epidermal growth factor receptor in non-small cell lung cancer - search and destroy. Eur J Cancer 42, 17-23

35. Sequist LV, Martins RG, Spigel D et al (2008) First-line gefitinib in patients with advanced non-small-cell lung cancer harboring somatic EGFR mutations. J Clin Oncol 26, 2442-2449

36. Francis JM, Zhang CZ, Maire CL et al (2014) EGFR variant 
heterogeneity in glioblastoma resolved through singlenucleus sequencing. Cancer Discov 4, 956-971

37. Cho J, Kim S, Du J and Meyerson M (2018) Autophosphorylation of the carboxyl-terminal domain is not required for oncogenic transformation by lung-cancer derived EGFR mutants. Int J Cancer 143, 679-685

38. Wang Z (2017) ErbB receptors and cancer. Methods Mol Biol 1652, 3-35

39. Sorkin A and Goh LK (2009) Endocytosis and intracellular trafficking of ErbBs. Exp Cell Res 315, 683-696

40. Segatto O, Anastasi S and Alema S (2011) Regulation of epidermal growth factor receptor signalling by inducible feedback inhibitors. J Cell Sci 124, 1785-1793

41. Anastasi S, Lamberti D, Alema S and Segatto O (2016) Regulation of the ErbB network by the MIG6 feedback loop in physiology, tumor suppression and responses to oncogene-targeted therapeutics. Semin Cell Dev Biol 50, 115-124

42. Anastasi S, Fiorentino L, Fiorini M et al (2003) Feedback inhibition by RALT controls signal output by the ErbB network. Oncogene 22, 4221-4234

43. Hackel PO, Gishizky M and Ullrich A (2001) Mig-6 is a negative regulator of the epidermal growth factor receptor signal. Biol Chem 382, 1649-1662

44. Anastasi S, Baietti MF, Frosi Y, Alema $S$ and Segatto O (2007) The evolutionarily conserved EBR module of RALT/MIG6 mediates suppression of the EGFR catalytic activity. Oncogene 26, 7833-7846

45. Zhang X, Pickin KA, Bose R, Jura N, Cole PA and Kuriyan J (2007) Inhibition of the EGF receptor by binding of MIG6 to an activating kinase domain interface. Nature 450, 741-744

46. Park E, Kim N, Ficarro SB et al (2015) Structure and mechanism of activity-based inhibition of the EGF receptor by Mig6. Nat Struct Mol Biol 22, 703-711

47. Ying $H$, Zheng $H$, Scott $K$ et al (2010) Mig-6 controls EGFR trafficking and suppresses gliomagenesis. Proc Natl Acad Sci U S A 107, 6912-6917

48. Frosi Y, Anastasi S, Ballaro C et al (2010) A two-tiered mechanism of EGFR inhibition by RALT/MIG6 via kinase suppression and receptor degradation. J Cell Biol 189, 557-571

49. Ferby I, Reschke M, Kudlacek O et al (2006) Mig6 is a negative regulator of EGF receptor-mediated skin morphogenesis and tumor formation. Nat Med 12, 568-573

50. Broadbridge VT, Karapetis CS and Price TJ (2012) Cetuximab in metastatic colorectal cancer. Expert Rev Anticancer Ther 12, 555-565

51. Dokala A and Thakur SS (2017) Extracellular region of epidermal growth factor receptor: a potential target for anti-EGFR drug discovery. Oncogene 36, 2337-2344

52. Valley CC, Arndt-Jovin DJ, Karedla $N$ et al (2015) Enhanced dimerization drives ligand-independent activity of mutant epidermal growth factor receptor in lung cancer. Mol Biol Cell 26, 4087-4099

53. Maity TK, Venugopalan A, Linnoila I et al (2015) Loss of MIG6 accelerates initiation and progression of mutant epidermal growth factor receptor-driven lung adenocarcinoma. Cancer Discov 5, 534-549

54. Westover D, Zugazagoitia J, Cho BC, Lovly CM and
Paz-Ares L (2018) Mechanisms of acquired resistance to first- and second-generation EGFR tyrosine kinase inhibitors. Ann Oncol 29, i10-i19

55. Tan CS, Kumarakulasinghe NB, Huang YQ et al (2018) Third generation EGFR TKIs: current data and future directions. Mol Cancer 17, 29

56. Wang S, Tsui ST, Liu C, Song Y and Liu D (2016) EGFR C797S mutation mediates resistance to third-generation inhibitors in T790M-positive non-small cell lung cancer. J Hematol Oncol 9, 59

57. Gerhart J, Thevenin AF, Bloch E, King KE and Thevenin D (2018) Inhibiting epidermal growth factor receptor dimerization and signaling through targeted delivery of a juxtamembrane domain peptide mimic. ACS Chem Biol 13, 2623-2632

58. Li N and Wei M (2017) Conversion of MIG6 peptide from the nonbinder to binder of lung cancer-related EGFR by phosphorylation and cyclization. Artif Cells Nanomed Biotechnol 45, 1023-1028

59. Yu XD, Yang R and Leng CJ (2016) Truncation, modification, and optimization of MIG6(segment 2) peptide to target lung cancer-related EGFR. Comput Biol Chem 61, 251-257

60. Frederick L, Wang XY, Eley G and James CD (2000) Diversity and frequency of epidermal growth factor receptor mutations in human glioblastomas. Cancer Res 60, 13831387

61. Barber TD, Vogelstein B, Kinzler KW and Velculescu VE (2004) Somatic mutations of EGFR in colorectal cancers and glioblastomas. N Engl J Med 351, 2883

62. Yamazaki H, Fukui Y, Ueyama Y et al (1988) Amplification of the structurally and functionally altered epidermal growth factor receptor gene (c-erbB) in human brain tumors. Mol Cell Biol 8, 1816-1820

63. Huang SF, Liu HP, Li LH et al (2004) High frequency of epidermal growth factor receptor mutations with complex patterns in non-small cell lung cancers related to gefitinib responsiveness in Taiwan. Clin Cancer Res 10, 8195-8203

64. Sasaki H, Okuda K, Takada M et al (2008) A novel EGFR mutation $\mathrm{D} 1012 \mathrm{H}$ and polymorphism at exon 25 in Japanese lung cancer. J Cancer Res Clin Oncol 134, 13711376

65. Balak MN, Gong Y, Riely GJ et al (2006) Novel D761Y and common secondary T790M mutations in epidermal growth factor receptor-mutant lung adenocarcinomas with acquired resistance to kinase inhibitors. Clin Cancer Res 12, 6494-6501

66. Asahina H, Yamazaki K, Kinoshita I, Yokouchi H, DosakaAkita $\mathrm{H}$ and Nishimura $\mathrm{M}$ (2006) Non-responsiveness to gefitinib in a patient with lung adenocarcinoma having rare EGFR mutations S768I and V769L. Lung Cancer 54, 419-422

67. Tokumo M, Toyooka S, Ichihara S et al (2006) Double mutation and gene copy number of EGFR in gefitinib refractory non-small-cell lung cancer. Lung Cancer 53, 117-121

68. Han SW, Kim TY, Hwang PG et al (2005) Predictive and prognostic impact of epidermal growth factor receptor mutation in non-small-cell lung cancer patients treated with gefitinib. J Clin Oncol 23, 2493-2501 
69. Montagut C, Dalmases A, Bellosillo B et al (2012) Identification of a mutation in the extracellular domain of the Epidermal Growth Factor Receptor conferring cetuximab resistance in colorectal cancer. Nat Med 18, 221-223

70. Kohsaka S, Nagano M, Ueno T et al (2017) A method of high-throughput functional evaluation of EGFR gene variants of unknown significance in cancer. Sci Transl Med 9, pii: eaan6566

71. Sanchez-Martin FJ, Bellosillo B, Gelabert-Baldrich M et al
(2016) The First-in-class Anti-EGFR antibody mixture sym004 overcomes cetuximab resistance mediated by EGFR extracellular domain mutations in colorectal cancer. Clin Cancer Res 22, 3260-3267

72. Arena S, Bellosillo B, Siravegna G et al (2015) Emergence of multiple EGFR extracellular mutations during cetuximab treatment in colorectal cancer. Clin Cancer Res 21, 21572166 